\title{
Influence of Sea Surface Roughness on the Electromagnetic Wave Propagation in the Duct Environment
}

\author{
Xiaofeng ZHAO, Sixun HUANG \\ Institute of Meteorology, PLA Univ. of Sci. \& Tech., Nanjing 211101, China \\ zxf_best@126.com, huangsxp@yahoo.com.cn
}

\begin{abstract}
This paper deals with a study of the influence of sea surface roughness on the electromagnetic wave propagation in the duct environment. The problem of electromagnetic wave propagation is modeled by using the parabolic equation method. The roughness of the sea surface is computed by modifying the smooth surface Fresnel reflection coefficient to account for the reduction in the specular reflection due to the roughness resulting from sea wind speed. The propagation model is solved by the mixed Fourier split-step algorithm. Numerical experiments indicate that wind-driven roughened sea surface has an impact on the electromagnetic wave propagation in the duct environment, and the strength is intensified along with the increment of sea wind speeds and/or the operating frequencies. In a fixed duct environment, however, proper disposition of the transmitter could reduce these impacts.
\end{abstract}

\section{Keywords}

Electromagnetic wave propagation, parabolic equation, sea wind speed, evaporation duct.

\section{Introduction}

Ducted propagation over the sea surface can seriously impact shipboard radar and communications, which is extremely important in telecommunications for the Navy. The physics of propagation is affected by ever-changing atmospheric conditions and by complex features on the sea surface [1]. Numerous numerical methods are available for predicting electromagnetic wave propagation such as geometric optics, normal mode analysis, parabolic equation (PE) method, and combinations of the above [2-5]. The most popular approach of the mentioned above is $\mathrm{PE}$ which could be used to predict electromagnetic wave propagation in a complex environment. Previously, use of the PE to investigate propagation in an inhomogeneous atmosphere and over irregular terrain has been published a lot [6-10]. However, the researches of the propagation over the rough sea surface are very few. In 2003, Guillet et al. employed $\mathrm{PE}$ to studied low grazing angle propagation above rough surface [11]. In this paper, we will employ Miller-BrownVegh model to study the influence of wind-driven roughness on the electromagnetic wave propagation in the evaporation duct environment. A detailed discussion will be focused on different numerical values for various important parameters, i.e. sea wind speed, evaporation duct height, operating frequency, and antenna altitude.

The remainder of this paper is organized as follows. In section 2, a simple description of the electromagnetic wave propagation model is introduced. Section 3 constructs the relationship between the rough sea surface and the smooth sea surface through the reflection coefficient. Finally, a discussion of simulations of different parameters is presented in section 4 .

\section{The Electromagnetic Wave Propagation Model}

For many years now PE has been used to model electromagnetic wave propagation in a complex environment. PE can be derived from the wave equation under certain assumptions, and it has the form [7],

$$
\frac{\partial^{2} u(x, z)}{\partial z^{2}}+2 i k_{0} \frac{\partial u(x, z)}{\partial x}+k_{0}^{2}\left[n^{2}(x, z)-1\right] u(x, z)=0
$$

where $u$ represents a scalar component of the electric field for horizontal polarization or a scalar component of the magnetic field for vertical polarization, $k_{0}$ is the free-space wave number, $x$ is the range axis, $z$ is the height axis, and $n$ is the index of refraction. Owing to $n$ is very close to unity, for environmental inputs, modified refractivity $M$ is used to describe the information of the atmospheric environment. $M$ is defined by

$$
M(x, z)=\left(n(x, z)-1+\frac{z}{a_{\mathrm{e}}}\right) \times 10^{6}
$$

where $a_{\mathrm{e}}$ is the radius of the earth.

The two most popular approaches to numerically solving PE are the use of implicit finite differences and the Fourier split-step algorithm. However, the latter is much 
more numerically efficient [3], and is adopted in our calculations. Generally, split-step PE solutions to electromagnetic problems implemented either Dirichlet or Neumann boundary conditions corresponding to horizontally or vertically polarized propagation, respectively, over a perfectly conduct surface. In rough sea surface, however, the perfectly conduct surface assumption is not sufficient and use of a surface impedance is highly desirable. The impedance boundary condition is

$$
\left.\partial_{z}(x, z)\right|_{z=0}+\alpha u(x, 0)=0
$$

and

$$
\alpha=i k_{0} \sin \theta\left(\frac{1-\Gamma}{1+\Gamma}\right)
$$

where $\Gamma$ is the Fresnel reflection coefficient and $\theta$ is the grazing angle measured from the local surface tangent, i.e. the complement of the incidence angle.

The mixed Fourier transform introduced as a rigorous method to incorporate surface impedance into split-step solution of the parabolic equation was described in detail in [4], and a fast computation algorithm for the mixed Fourier transform split-step solution of PE was presented in [12].

\section{Sea Surface Roughness Modeling}

Using the impedance boundary condition, the reflection coefficient $\Gamma$ should be determined. In terms of the law of reflection, for a giving grazing angle $\theta$, the smooth surface reflection coefficient $\Gamma_{S}$ for horizontal polarization and vertical polarization could, respectively, be described as [13]

$$
\Gamma_{S H}=\frac{\sin \theta-\sqrt{n_{s}^{2}-\cos ^{2} \theta}}{\sin \theta+\sqrt{n_{s}^{2}-\cos ^{2} \theta}}
$$

and

$$
\Gamma_{S V}=\frac{n_{s}^{2} \sin \theta-\sqrt{n_{s}^{2}-\cos ^{2} \theta}}{n_{s}^{2} \sin \theta+\sqrt{n_{s}^{2}-\cos ^{2} \theta}}
$$

where $n_{s}$ is the complex dielectric constants, defined by

$$
n_{s}=\sqrt{\varepsilon+i 60 \sigma \lambda} \text {. }
$$

Here, $\varepsilon$ and $\sigma$ are the relative permittivity and conductivity respectively, which are functions of radio frequency recommended by International Radio Consulting Committee (CCIR), and $\lambda$ is the electromagnetic wave length.

One approach to modeling sea surface roughness involves multiplying the smooth surface Fresnel reflection coefficients by a roughness parameter, which has been calculated by Ament [14] for a Gaussian distribution of heights and corrected by Miller et al. [15]. The rough surface reflection coefficient, $\Gamma_{R}$, is taken as

$$
\Gamma_{R}=\Gamma_{S} \cdot \exp \left[-2(2 \pi g)^{2}\right] \cdot I_{0}\left[2(2 \pi g)^{2}\right]
$$

and

$$
g=\frac{\sigma_{h} \sin \theta}{\lambda}
$$

where $I_{0}(x)$ is the modified Bessel function $J_{0}(i x)$, and $\sigma_{h}$ is the standard deviation of the sea surface elevation determined from wind speed, $\mu(\mathrm{m} / \mathrm{s})$, by

$$
\sigma_{h}=0.0051 \mu^{2} \text {. }
$$

As mentioned above, when modifying reflection coefficients using the Miller-Brown-Vegh model, the resulting expression for $\alpha$ has a strong dependence on the local grazing angle $\theta$ which is, in general, a function of geometry and, more importantly, atmospheric refractivity. Dockery and Kuttler have pointed out that geometric optics (GO) could provide a qualified grazing angle estimate in the evaporation duct environment [12]. Therefore, GO will be adopted in our computation.

\section{Numerical Experiments}

The purpose of this paper is to study the influence of sea surface roughness to the electromagnetic wave propagation in the evaporation duct environment. First, an evaporation duct model should be selected. For thermally neutral conditions in which the air and sea temperature are equal, the modified refractivity $M$ can be determined at any height $z$ by the relationship [16]

$M(z)=M(0)+0.125 z-0.125 d \ln \left[\left(z+z_{0}\right) / z_{0}\right]$

where $d$ is evaporation duct height, $\ln [\cdot]$ is the natural logarithm, and $z_{0}$ is momentum roughness length of $1.5 \times 10^{-4} \mathrm{~m}$.

In the following, two experiments are performed. For every experiment, a Gaussian antenna pattern is used and horizontal polarization is assumed. The elevation angle is $0 \mathrm{deg}$ and the beamwidth is $1 \mathrm{deg}$. The detailed expression of the Gaussian antenna pattern could refer to [7].

Experiment 1: The evaporation duct height and the antenna altitude are fixed at $30 \mathrm{~m}$ and $10 \mathrm{~m}$, respectively. Then, the propagation characteristics at different sea wind speeds and operating frequencies are investigated. The sea wind speeds are changeable, from $0 \mathrm{~m} / \mathrm{s}$ to $10 \mathrm{~m} / \mathrm{s}$.

Using the PE/mixed Fourier split-step algorithm, the value of one-way propagation loss is computed. Here, the computation domain is fixed on $[100 \mathrm{~km} \times 200 \mathrm{~m}]$, and the horizontal increment and vertical increment are $500 \mathrm{~m}$ and $1 \mathrm{~m}$, respectively. The coverage diagram $(\mathrm{dB})$ for one-way propagation loss is shown in Fig. 1, in which the operating frequency is $8000 \mathrm{MHz}$, Fig. 1(a) is for sea wind speed of $0 \mathrm{~m} / \mathrm{s}$, Fig. 1(b) is for $10 \mathrm{~m} / \mathrm{s}$, and Fig. 1(c) is the absolute difference of the above two figures. 

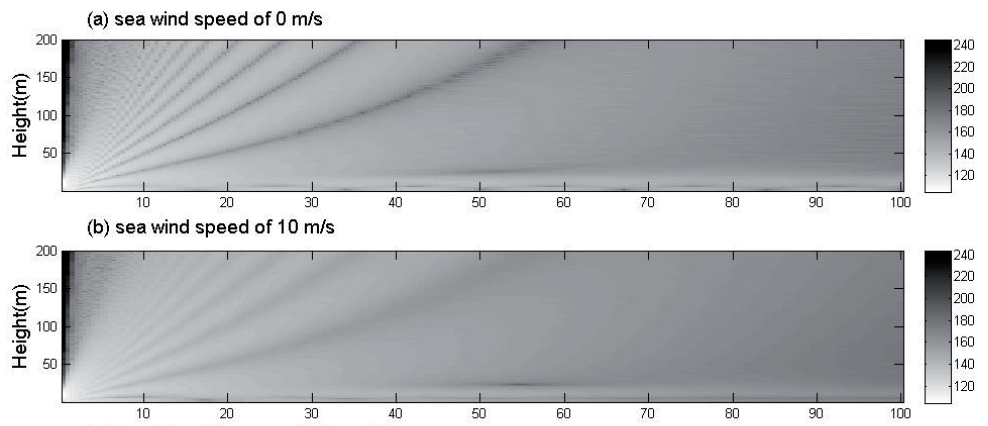

(c) absolute difference of (a) and (b)

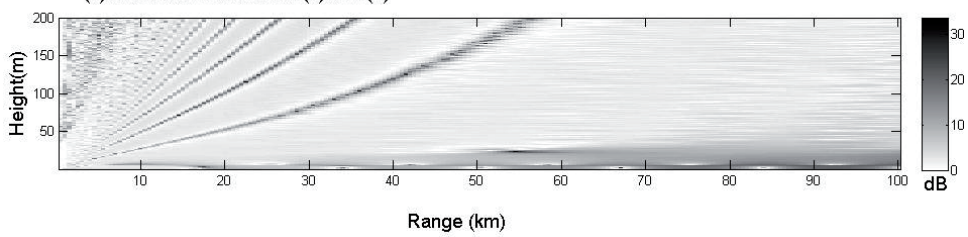

Fig. 1. The coverage diagram (dB) for one-way propagation loss. (a) is for $0 \mathrm{~m} / \mathrm{s}$; (b) is for $10 \mathrm{~m} / \mathrm{s}$, (c) is the absolute difference of (a) and (b).

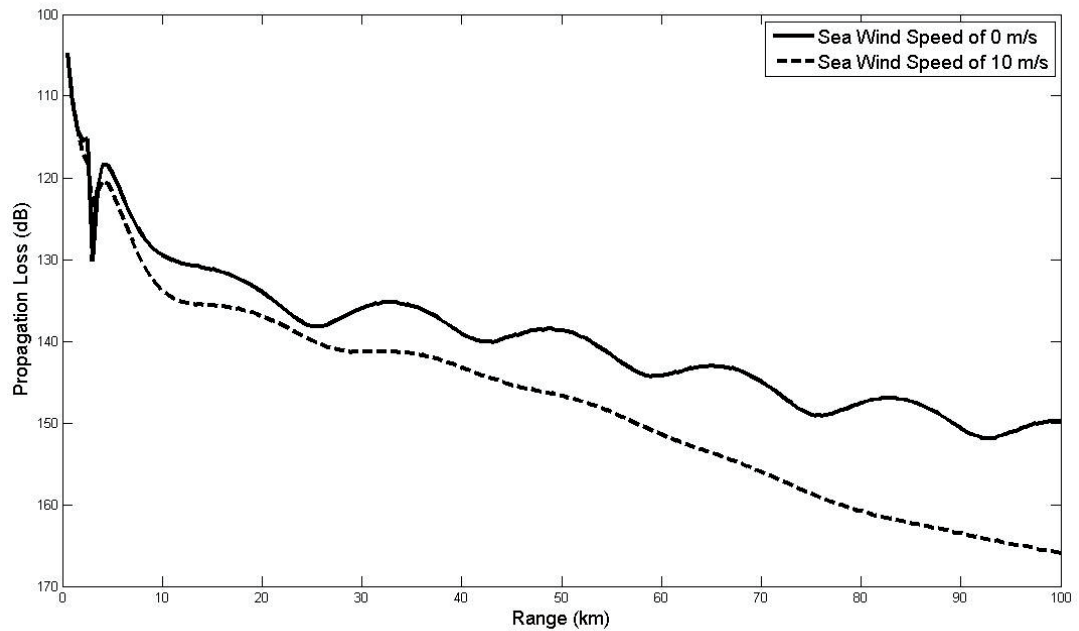

Fig. 2. Comparison of propagation loss at antenna altitude $(10 \mathrm{~m})$ in a $30 \mathrm{~m}$ evaporation duct between smooth sea surface (sea wind speed of $0 \mathrm{~m} / \mathrm{s}$, solid line) and rough sea surface (sea wind speed of $10 \mathrm{~m} / \mathrm{s}$, dashed line). The operating frequency is $8000 \mathrm{MHz}$.

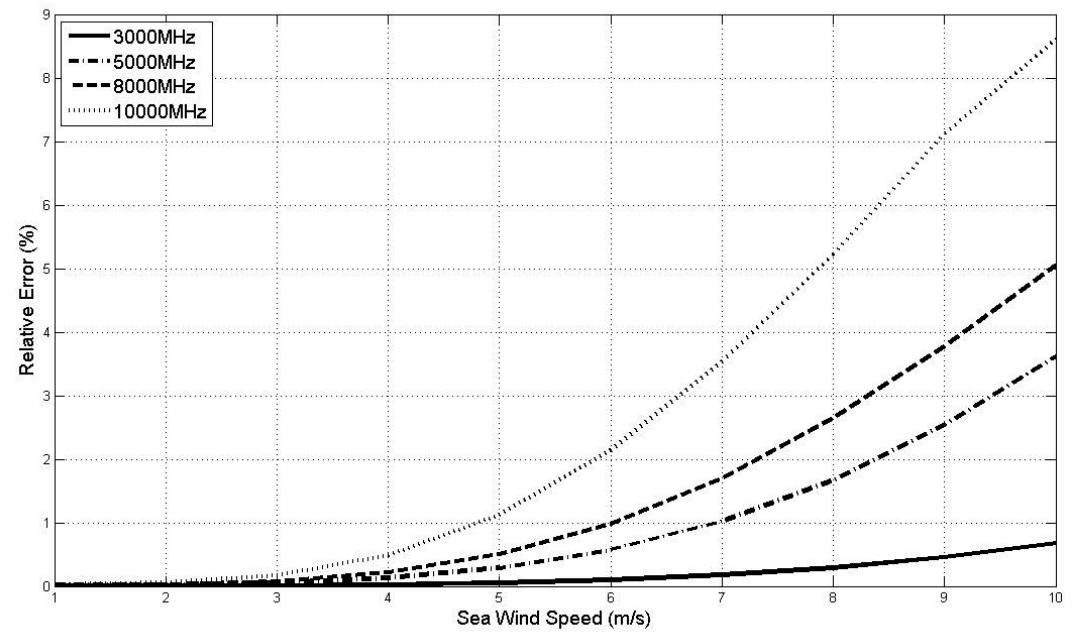

Fig. 3. Relative errors at different operating frequencies and different sea wind speeds. 
In order to analyze the influence of wind-driven roughness on the electromagnetic wave propagation, the values of propagation loss at the antenna altitude are selected to compute the relative error of the propagation loss between the smooth sea surface and the rough sea surface. The relative error $\Lambda$ is defined as

$$
\Lambda=\frac{1}{N} \sum_{i=1}^{N}\left|\frac{P_{L i}^{u}-P_{L i}^{0}}{P_{L i}^{0}}\right| \times 100 \%
$$

where $P_{L}{ }^{u}$ is the value of propagation loss corresponding to the wind-driven rough sea surface and $P_{L}{ }^{0}$ corresponding to smooth surface, $N$ is the number of discrete points in the horizontal direction. The values of propagation loss along the horizontal distance at the antenna altitude $10 \mathrm{~m}$ in Fig. 1(a) and 1(b) are presented in Fig. 2. Using equation (12), the relative errors at different operating frequencies and different sea wind speeds are shown in Fig. 3.
From Fig. 1-3, it is clearly seen that, considering the sea surface roughness, the electromagnetic propagation loses more energy than the smooth surface case, and the propagation losses are strengthening with the distance. Fig. 3 shows that with the increment of sea wind speed and/or operating frequency, the relative errors between smooth sea surface and rough sea surface become larger and larger. In $10000 \mathrm{MHz}$ and $10 \mathrm{~m} / \mathrm{s}$ case, the relative error could even exceed $8.7 \%$. However, when the sea wind speed is under $5 \mathrm{~m} / \mathrm{s}$, the errors could be limited within $1.2 \%$.

Experiment 2: The operating frequency is fixed at $8000 \mathrm{MHz}$. The relationship between evaporation duct height and antenna altitude will be studied. The evaporation duct heights are set at $10 \mathrm{~m}, 20 \mathrm{~m}$, and $30 \mathrm{~m}$. Within the duct, the antenna altitudes are changeable, from $1 \mathrm{~m}$ to the duct height. Fig. 4 shows the relative error between smooth surface case (sea wind speed of $0 \mathrm{~m} / \mathrm{s}$ ) and rough surface case (sea wind speed of $10 \mathrm{~m} / \mathrm{s}$ ).

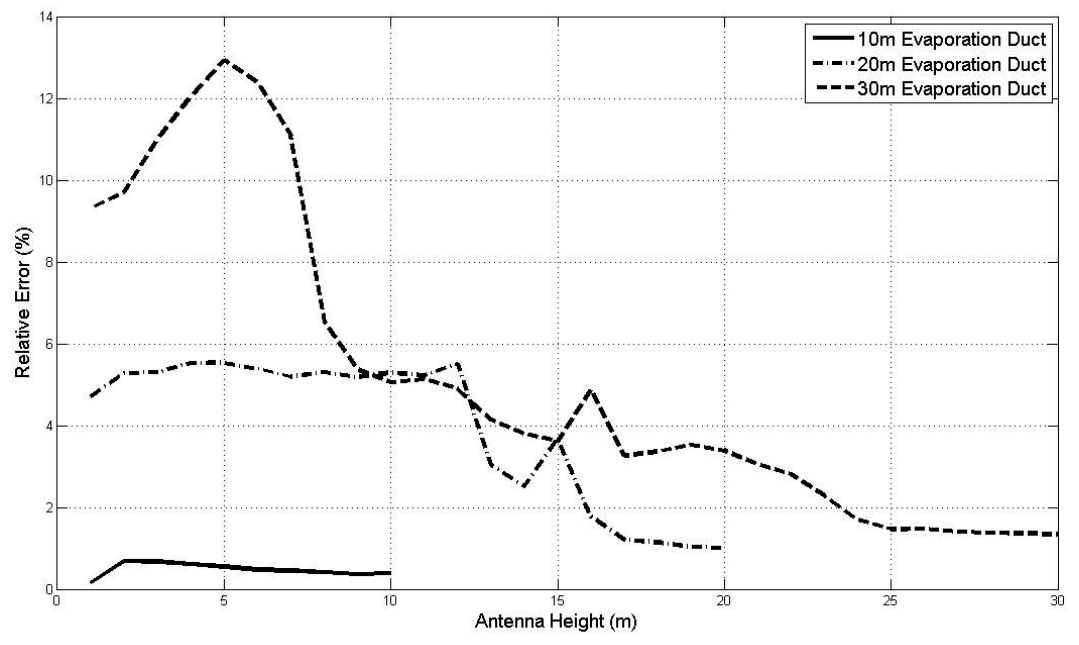

Fig. 4. Relative error between smooth surface case (sea wind speed of $0 \mathrm{~m} / \mathrm{s}$ ) and rough surface case (sea wind speed of $10 \mathrm{~m} / \mathrm{s}$ ) at different evaporation duct heights and different antenna altitudes.

From Fig. 4, we could see the position of antenna altitude within the duct has great impact upon the propagation loss in the rough sea surface case. The stronger the evaporation duct exists, the larger the relative error of propagation loss will be generated. In $10 \mathrm{~m}$ duct, the maximum error presents at transmitter altitude of $2 \mathrm{~m}$, in $20 \mathrm{~m}$ duct, the maximum error presents at $12 \mathrm{~m}$, and $5 \mathrm{~m}$ in $30 \mathrm{~m}$ evaporation duct. When the relative error reaches the maximum value, it will have a tendency to decreasing with the increment of the antenna altitude.

The above two experiments show that, in the evaporation duct environment, the electromagnetic wave propagation characteristics over the rough sea surface are different from that over the smooth sea surface. In general, the errors enlarge with the increment of sea wind speeds, operating frequencies and evaporation duct height. At a fixed atmosphere environment, proper disposition of the transmitter could reduce these errors. The minimum error value usually exists near the top of the duct. However, if the transmitter is setting at the top of the duct, it is not in favor of increasing radar detecting distance. In our simulations, the maximum sea wind speed is selected as $10 \mathrm{~m} / \mathrm{s}$. In practical duct environment, the sea wind speed is usually below $10 \mathrm{~m} / \mathrm{s}$ because larger wind speed is destructive to the generation of the evaporation duct. If the sea wind speed is not very large (under $5 \mathrm{~m} / \mathrm{s}$ ), we could ignore the influence of the sea surface roughness to the electromagnetic wave propagation in the duct environment.

\section{Conclusion}

This paper has investigated the influence of the sea surface roughness on the electromagnetic wave propagation in the evaporation duct environment. Considering the sea surface roughness, the impedance boundary condition was used, and the Miller-Brown-Vegh model was em- 
ployed to compute the wind-driven rough surface reflection coefficient. Two numerical experiments were performed in terms of different sea wind speeds, operating frequencies, evaporation duct heights and antenna altitudes, and present a straightforward understanding of the relationship of these parameters to the roughness influence on propagation characteristics. However, the Miller-Brown-Vegh model doesn't take into account the influence of the surface geometry, which will be investigated in our future work.

\section{Acknowledgements}

This work has been supported by the National Natural Science Foundation of China, grant No. 40775023, "Refractivity estimation from radar sea clutter by incorporating regularization techniques into data assimilation method".

\section{References}

[1] YAN, H. J., FU, Y., HONG, Z. J. Introduction to Modern Atmospheric Refraction. Shanghai: Science and Educational Press, 2006.

[2] HE, G. Y., LU, C. C., HONG, J. C., DENG, H. Computation and Measurement of Electromagnetic Scattering. Peking: National Defence Industry Press, 2006.

[3] VALTR, P., PECHAC, P. Tropospheric refraction modeling using ray-tracing and parabolic equation. Radioengineering, 2005, vol. 14 , no. 4 , p. $98-104$.

[4] KUTTLER, J. R., DOCKERY, G. D. Theoretical description of the parabolic approximation/ Fourier split-step method of representing electromagnetic propagation in the troposphere. Radio Science, 1991, vol. 26, no. 2, p. 381-393.

[5] HITNEY, H. V. Hybrid ray optics and parabolic equation methods for radar propagation modelling. In Proc. IEE Int. Conf. Radar. Brighton (U.K.), 1992, p. 58-61.

[6] BARRIOS, A. E. Parabolic equation modelling in horizontally inhomogeneous environments. IEEE Transactions on Antennas and Propagation, 1992, vol. 40, no. 7, p. 791-797.

[7] BARRIOS, A. E. A terrain parabolic equation model for propagation in the troposphere. IEEE Transactions on Antennas and Propagation, 1994, vol. 42, no. 1, p. 90-98.

[8] GRABNER, M, KVICERA, V. Clear-air propagation modelling using parabolic equation method. Radioengineering, 2003, vol. 12, no. 4 , p. $50-54$.
[9] OZGUN, O. Recursive two-way parabolic equation approach for modelling terrain effects in tropospheric propagation. IEEE Transactions on Antennas and Propagation, 2009, vol. 57, no. 9, p. 2706-2714.

[10] VALTR, P., PECHAC, P., KVICERA, V., GRABNER, M. A terrestrial multiple-receiver radio link experiment at $10.7 \mathrm{GHz}-$ comparisons of results with parabolic equation calculations. Radioengineering, 2010, vol. 19, no. 1, p. 117-121.

[11] GUillet, N., FABBro, V., BOURliER, C., COMBES, P. F. Low grazing angle propagation above rough surface by the parabolic wave equation. In Geoscience and Remote Sensing Symposium, 2003.

[12] DOCKERY, G. D., KUTTLER, J. R. An improved impedanceboundary algorithm for Fourier split-step solution of the parabolic wave equation. IEEE Transactions on Antennas and Propagation, 1996, vol. 44, no. 12. p. 1592-1599.

[13] YANG, X. Q., ZHAO, J. S., WANG, Y. Electromagnetic Field and Electromagnetic Wave. Peking: National Defence Industry Press, 2002.

[14] AMENT, W. S. Toward a theory of reflection by a rough surface. In Proc. IRE. 1953, vol. 41, p. 142-146.

[15] MILLER, A. R., BROWN, R. M., VEGH, E. New derivation for rough surface reflection coefficient and for the distribution of seawave elevations. In IEE Proc, 1984, vol. 131, no. 2, p. 114-116.

[16] HITNEY, H. V., VIETH, R. Statistical assessment of evaporation duct propagation. IEEE Transactions on Antennas and Propagation, 1990, vol. 38, no. 6, p. 794-799.

\section{About Authors ...}

Xiaofeng ZHAO was born in Jiangsu, China. He received his M.Sc. degree from PLA University of Science and Technology in 2009. Now he is working towards his Ph.D. at the Institute of Meteorology of the same university. His research interests include radiowave propagation modeling and atmospheric refractivity inversion from radar sea clutter.

Sixun HUANG was born in Shanghai, China. He received his M.Sc. degree in applied mathematics from Fundan University in 1981. He is now a Professor at the Institute of Meteorology, PLA University of Science and Technology. His research interests are in the field of radiowave propagation modeling and remote sensing technologies. 